\title{
Estimation of Systematic Errors of MODIS Thermal Infrared Bands
}

\author{
Ronggao G. Liu, Jiyuan Y. Liu, and Shunlin Liang, Senior Member, IEEE
}

\begin{abstract}
This letter reports a statistical method to estimate detector-dependent systematic error in Moderate Resolution Imaging Spectroradiometer (MODIS) thermal infrared (TIR) Bands 20-25 and 27-36. There exist scan-to-scan overlapped pixels in MODIS data. By analyzing a sufficiently large amount of those most overlapped pixels, the systematic error of each detector in the TIR bands can be estimated. The results show that the Aqua MODIS data are generally better than the Terra MODIS data in 160 MODIS TIR detectors. There are no detector-dependent systematic errors in Bands 31 and 32 for both Terra and Aqua MODIS data. The maximum detector errors are 3.00 $\mathrm{K}$ in Band 21 of Terra and $-8.15 \mathrm{~K}$ in that of Aqua for brightness temperatures of more than $250 \mathrm{~K}$.
\end{abstract}

Index Terms-Land surface temperature (LST), Moderate Resolution Imaging Spectroradiometer (MODIS), systematic errors, thermal infrared (TIR).

\section{INTRODUCTION}

$\mathbf{T}$ HE UNDERSTANDING of temporal and spatial distribution patterns of land surface temperature (LST) is important for climate, ecology, and hydrology studies. Remote sensing is the only effective means to achieve this goal [1]. The sea surface temperature retrieved from the Advanced Very HighResolution Radiometer (AVHRR) has been used extensively [2], but its calibration accuracy and spectral band range are limited. The radiance calibration of two Moderate Resolution Imaging Spectroradiometer (MODIS) sensors onboard Terra and Aqua satellites launched in 1999 and 2002 has been significantly improved. The MODIS LST accuracy is better than $1 \mathrm{~K}$ in the range from 263 to $300 \mathrm{~K}$ over most regions and is a few kelvin lower than the in situ measured LST in semiarid and arid regions [3]. However, systematic errors from channel detectors can reduce their performance. Although these errors had been measured and calibrated in a laboratory before launch [4], the small change in the optical status of MODIS instruments from voltage change, gas leak, and other factors may cause new biases. Detection of the systematic errors in different channels can help to reduce the uncertainty for retrieval of LST,

Manuscript received December 13, 2005; revised March 24, 2006. This work was supported in part by the National 973 Program under Grant 2002CB4125 and in part by the National Natural Science Foundation of China under Grant 40471098.

R. G. Liu and J. Y. Liu are with the State Key Laboratory of Resources and Environmental Information System, Institute of Geographical Sciences and Natural Resources Research, Chinese Academy of Sciences, Beijing 100101, China (e-mail: liurg@lreis.ac.cn).

S. Liang is with Department of Geography, University of Maryland, College Park, MD 20742 USA (e-mail: sliang@geog.umd.edu).

Digital Object Identifier 10.1109/LGRS.2006.879104 sea surface temperature, air temperature, and atmospheric water vapor [5].

Wan [5] has analyzed the channel systematic errors in Terra MODIS data. Several regions, in which the atmospheric conditions and land surface status are uniform, are selected as test sites. Because the observed targets are similar for different detectors, the discrepancies of different channels in the same band are mainly from channel systematic errors and noise. Band 31 was used to find the uniform region, and 14 sample sites were selected for data analyses from ten global regions. Each sample site consists of ten lines with 16 pixels per line, which correspond to the ten channels of MODIS thermal infrared (TIR) bands. The channel-dependent errors were estimated from statistical analyses of these data.

In this letter, we propose a new statistical method to estimate channel systematic errors, which does not require the uniform region as Wan's method does. There exist some most overlapped pixel pairs in two consecutive scans. Since each pixel pair observes nearly the same atmosphere-Earth target, their measured bright temperatures should be equal if no noise or detector systematic errors exist. If we assume that the actual observational differences of the most overlapped pixel pairs are from the detectors' systematic errors and random noises, the channel systematic errors can be estimated by statistically analyzing a large amount of these data.

\section{DATA AND MEthod}

\section{A. Data}

MODIS data consist of 36 bands with a maximum spatial resolution of $250 \mathrm{~m}$ from the cross-track scanning of multielement linear detector array. Each band for resolutions of $1 \mathrm{~km}, 500 \mathrm{~m}$, and $250 \mathrm{~m}$ has 10,20 , and 40 detectors aligned in the along-track direction, respectively. The 16 TIR bands for detecting temperature are from 3 to $15 \mu \mathrm{m}$ and $1-\mathrm{km}$ resolution at the nadir. For each scanning, the satellite travels $10 \mathrm{~km}$, the double-sided scan mirror sweeps out a $110^{\circ}$ Earth field of view (equivalent of $2340 \mathrm{~km}$ ), and each TIR detector takes 1354 samples. Thus, the 1354 columns and ten rows of data can be acquired by ten TIR detectors during this operation. In this letter, a channel is defined as one line of data that are captured by the same detector with one scanning. Scan data are those captured by all detectors with one scanning. One scan constitutes 10,20 , or 40 channels according to the band resolution. One band data comprise a set of scans. For example, each TIR band scan consists of ten channels, and 5-min granule data include 203 scans for each band. Because 
of the Earth's curvature, pixel resolution decreases but the pixel footprint expands while away from the nadir. While the view angle reaches a certain degree, some Earth targets could be observed twice by different detectors of the same band in two consecutive scans. For instance, in two adjacent scans of 1-km band data, two pixels overlap with a view angle of about $25^{\circ}$ and half of the pixels do at maximum view angle of $55^{\circ}$. This causes a panoramic "bowtie" effect in MODIS data.

\section{B. Method}

For each pixel, its brightness temperature can be defined as

$$
\begin{aligned}
T_{\mathrm{obs}}(b, c, i)=T_{\mathrm{BT}}(b, i)+ & \delta T_{\text {mirror }}(b, i \% 2) \\
+ & \delta T_{\mathrm{sys}}(b, c)+\delta T_{\text {noise }}(b, c, i)
\end{aligned}
$$

where band $b=20-25,27-36$; channel $c=1-10 ; i$ is the scan counter; $i \% 2$ is the modulus of $i$ and 2 , which means the mirror side, and we assume that its value 0 represents the A side and 1 as the $\mathrm{B}$ side; $T_{\text {obs }}$ is the observation value by satellite detector in kelvin; $T_{\mathrm{BT}}$ is the top-of-atmosphere (TOA) brightness temperature; $\delta T_{\text {mirror }}$ is the error from different mirror sides; $\delta T_{\mathrm{sys}}$ is the systematic error; and $\delta T_{\text {noise }}$ is the random noise.

In same band, the observational brightness temperature difference between the pixel in channel $c_{1}$ of scan $i$ and that in channel $c_{2}$ in scan $i+1$ is

$$
\begin{aligned}
T_{\mathrm{obs}} & \left(b, c_{1}, i\right)-T_{\mathrm{obs}}\left(b, c_{2}, i+1\right) \\
= & {\left[T_{\mathrm{BT}}(b, i)-T_{\mathrm{BT}}(b, i+1)\right] } \\
& +\left[\delta T_{\text {mirror }}(b,(i \% 2))-\delta T_{\text {mirror }}(b,(i+1) \% 2)\right] \\
& +\left[\delta T_{\mathrm{sys}}\left(b, c_{1}\right)-\delta T_{\mathrm{sys}}\left(b, c_{2}\right)\right] \\
& +\left[\delta T_{\text {noise }}\left(b, c_{1}, i+1\right)-\delta T_{\text {noise }}\left(b, c_{2}, i+1\right)\right] .
\end{aligned}
$$

The summation of $N$ scans is

$$
\begin{aligned}
\sum_{i=1}^{N} & {\left[T_{\mathrm{obs}}\left(b, c_{1}, i\right)-T_{\mathrm{obs}}\left(b, c_{2}, i+1\right)\right] } \\
= & \sum_{i=1}^{N}\left[T_{\mathrm{BT}}(b, i)-T_{\mathrm{BT}}(b, i+1)\right] \\
& +\sum_{i=1}^{N}\left[\delta T_{\text {mirror }}(b, i \% 2)-\delta T_{\text {mirror }}(b,(i+1) \% 2)\right] \\
& +\sum_{i=1}^{N}\left[\delta T_{\text {sys }}\left(b, c_{1}\right)-\delta T_{\text {sys }}\left(b, c_{2}\right)\right] \\
& +\sum_{i=1}^{N}\left[\delta T_{\text {noise }}\left(b, c_{1}, i\right)-\delta T_{\text {noise }}\left(b, c_{2}, i+1\right)\right] .
\end{aligned}
$$

We assume that the TOA brightness temperature difference between the two most overlapped pixels in channel $c_{1}$ of scan $i$ and channel $c_{2}$ of scan $i+1$ is small, and that this difference distributes randomly so that it can be treated as random noise. When $N$ is large enough, the summation of random noise is 0 , i.e.,

$$
\begin{aligned}
\sum_{i=1}^{N}\left[T_{\mathrm{BT}}(b, i)-T_{\mathrm{BT}}(b, i+1)\right] & =0 \quad(N \rightarrow \infty) \\
\sum_{i=1}^{N} \delta T_{\text {noise }}\left(b, c_{1}, i\right) & =0 \quad(N \rightarrow \infty) \\
\sum_{i=1}^{N} \delta T_{\text {noise }}\left(b, c_{2}, i+1\right) & =0 \quad(N \rightarrow \infty) .
\end{aligned}
$$

The mirror sides A and B being alternately used for scanning, their effects can be cancelled out while $N$ is even. If $N$ is odd, this effect can also be ignored while $N$ is large enough, i.e.,

$\frac{1}{N} \sum_{i=1}^{N}\left[\delta T_{\text {mirror }}(b, i \% 2)-\delta T_{\text {mirror }}(b,(i+1) \% 2)\right]=0(N \rightarrow \infty)$.

Because the systematic error for the same detector is equal, when $N \rightarrow \infty$, it follows that

$$
\begin{aligned}
\delta T_{\mathrm{sys}}\left(b, c_{1}\right)- & \delta T_{\mathrm{sys}}\left(b, c_{2}\right)=\frac{1}{N} \\
& \times \sum_{i=1}^{N}\left[T_{\mathrm{obs}}\left(b, c_{1}, i\right)-T_{\mathrm{obs}}\left(b, c_{2}, i+1\right)\right] .
\end{aligned}
$$

Mark the ten channels from 1 to 10 in sequence. Channels 6-10 of one scan overlap with Channels 1-5 of its consecutive scan at a certain view angle. Since two consecutive scans overlap from none in the middle to half on the edge, the most overlapped channel pixel pairs can be $(10,1),(9,1),(10,2),(8,1),(9,2)$, $(10,3),(7,1),(8,2),(9,3),(10,4),(6,1),(7,2),(8,3)$, $(9,4)$, and $(10,5)$. From $(8)$, a set of 15 equations can be established for the ten channel error variables. However, this set of equations is singular from which the ten variables cannot be solved. At least one constraint should be added to it for obtaining the solutions.

Since one detector's systematic error is a deviation from the average value of all detectors, the summation of ten detector errors should be zero. It follows that

$$
\sum_{c=1}^{10} \delta T_{\mathrm{sys}}(b, c)=0 .
$$

The optimized solution of these ten variables can be determined from the 16 equations. However, the relative nonoverlapped area in pairs $(10,1),(10,2),(9,1),(10,3),(9,2)$, and $(8,1)$ is larger than others in the cross-track direction, so these pairs were deleted from equations. Thus, systematic errors of the ten detectors $\delta T_{\mathrm{sys}}(b, c)(c=1, \ldots, 10)$ can be calculated from ten linear equations.

\section{Location of the Most Overlapped Pixels of Two Scans}

The most overlapped pixel position can be estimated based on the satellite orbiting altitude and the Earth's radius. We define the Earth radius as $R$, the satellite orbiting altitude as 
$h$, the view angle as $\varphi$, the pixel resolution at the nadir as $\mathrm{Re}$, and the pixel width in track direction with view angle $\varphi$ as $D$. The following equation can be established:

$\mathrm{D}(\varphi)=\operatorname{Re} / h \bullet\left[H * \cos (\varphi)-\sqrt{\left(R^{2}-H^{2} \sin ^{2}(\varphi)\right)}\right]$

where $H=h+R$. From (10), the pixel size $D$ 's for the overlapped pairs amount from five to one in two consecutive scans are $1.11,1.25,1.43,1.67$, and $2.0 \mathrm{~km}$, respectively. The view angles for them are $24.5^{\circ}, 34.7^{\circ}, 42.6^{\circ}, 49.2^{\circ}$, and $55.0^{\circ}$, which correspond to sample pixel positions $377,251,154,71$, and 1 .

Position can also be estimated from the analysis of MODIS data directly. From the assumption above, the difference of the most overlapped pixel pairs should be minimized in all pixel pairs. The absolutes of brightness temperature differences of 1354 pixel pairs for $N$ scans ( $N$ is large enough) and their average were calculated. Those pixels with minimum average differences can be considered as the most overlapped pixels. Among the 16 TIR bands, the channel errors of Bands 31 and 32 are basically $0 \mathrm{~K}$ (see Section III). Thus, Band 31 is used to find the position of the most overlapped pixels in two scans. The MODIS data were collected at the Beijing MODIS receiving station in February, May, August, and December of 2002. The most overlapped position in two scans is found at pixel 1353 for $(10,5),(9,4),(8,3),(7,2)$, and $(6,1)$; at pixel 1288 for $(10,4),(9,3),(8,2)$, and $(7,1)$; at pixel 1208 for $(10,3)$, $(9,2)$, and $(8,1)$; at pixel 1119 for $(10,2)$ and $(9,1)$; and at pixel 999 for $(10,1)$. The location is symmetric around the nadir for different pairs. It can be found that the positions are similar for the same pair group, such as those for $(10,5)$ and $(9,4)$, which are equal. This demonstrates that there indeed exist the most overlapped pixels in two consecutive scans. Band 32 has very similar results with Band 31, which proves that the locations of the most overlapped pixels are stable in different bands. These positions can be compared with the pixel positions from theoretical estimation. The position differences are one, five, eight, 16, and 22 for the overlapped pairs amount from five to one. This also shows that the positions of the most overlapped pixel pairs from two different methods are well consistent.

\section{RESUlts AND Discussion}

\section{A. Errors of Terra MODIS Detector in 2002}

MODIS data collected at the Beijing receiving station in February, March, August, and December of 2002, which represent four different seasons, are chosen for the analysis. All invalid data were excluded, and the clouds were masked. We analyzed the data of four months, separately and as a whole, and the results are quite similar. The results from the combination of the four months are shown in Table I. The channel errors are very small in Bands 31 and 32, which are nearly zero. These two bands are extensively used in surface temperature retrievals in most split-window methods, in which the uncertainties from the instruments can be ignored. The channel systematic errors are relatively large in Bands 21, 25, 27, 28, and 36. The three largest errors occur in Channels 9 and 1 of Band 21 and Channel 2 of Band 28. Band 21 is frequently used to detect hot spots.
TABLE I

DETECTOR SySTEMATIC ERRORS FOR TERRA MODIS (IN KELVIN)

\begin{tabular}{lrrrrrrrrrr}
\hline \hline Band & 1 & 2 & 3 & 4 & 5 & 6 & 7 & 8 & 9 & 10 \\
\hline \hline 20 & 0.08 & -0.01 & -0.01 & 0.00 & -0.04 & -0.06 & -0.01 & -0.01 & -0.01 & 0.06 \\
21 & -1.69 & 0.36 & 0.66 & -0.84 & -0.87 & -0.80 & 0.36 & 0.08 & 3.00 & -0.24 \\
22 & -0.01 & -0.01 & -0.04 & -0.05 & -0.04 & -0.02 & -0.01 & 0.24 & -0.04 & -0.01 \\
23 & 0.18 & -0.07 & -0.07 & -0.07 & -0.09 & -0.07 & -0.07 & -0.07 & -0.07 & 0.38 \\
24 & -0.62 & 0.11 & -0.12 & -0.01 & 0.08 & 0.19 & 0.11 & 0.13 & 0.05 & 0.08 \\
25 & 0.27 & -0.12 & -0.13 & 0.27 & -0.11 & -0.09 & -0.26 & -0.12 & -0.05 & 0.33 \\
27 & 0.29 & 0.27 & 0.24 & 0.21 & 0.06 & 0.61 & -0.23 & -0.37 & -0.73 & -0.35 \\
28 & -0.02 & 1.40 & 0.48 & -0.25 & -0.23 & -0.25 & -0.27 & -0.20 & -0.36 & -0.29 \\
29 & 0.26 & -0.03 & -0.03 & -0.03 & -0.05 & 0.00 & -0.03 & -0.03 & -0.03 & -0.03 \\
30 & -0.85 & -0.05 & 0.00 & -0.12 & 0.49 & -0.04 & -0.05 & 0.70 & -0.08 & 0.02 \\
31 & 0.00 & 0.00 & 0.00 & 0.00 & 0.00 & 0.00 & 0.00 & 0.00 & 0.00 & 0.00 \\
32 & 0.00 & 0.00 & 0.00 & 0.00 & 0.00 & 0.00 & 0.00 & 0.00 & 0.00 & 0.00 \\
33 & -0.36 & -0.03 & 0.05 & 0.02 & 0.01 & 0.04 & 0.02 & 0.05 & 0.08 & 0.10 \\
34 & 0.06 & 0.03 & 0.06 & 0.06 & -0.05 & 0.06 & -0.20 & -0.15 & 0.06 & 0.07 \\
35 & -0.14 & -0.02 & -0.02 & -0.02 & 0.07 & 0.21 & -0.02 & -0.02 & -0.02 & -0.02 \\
36 & -0.30 & -0.20 & -0.03 & -0.02 & 0.19 & 0.28 & 0.17 & -0.03 & -0.03 & -0.04 \\
\hline \hline
\end{tabular}

TABLE II

Detector Systematic ERRORS FOR AQUA MODIS (IN KELVIN)

\begin{tabular}{lrrrrrrrrrr}
\hline \hline Band & 1 & 2 & 3 & 4 & 5 & 6 & 7 & 8 & 9 & 10 \\
\hline \hline 20 & -0.17 & 0.00 & 0.00 & 0.05 & 0.05 & 0.01 & 0.00 & 0.00 & -0.01 & 0.05 \\
21 & 5.10 & -3.31 & -3.52 & 2.27 & -8.15 & 3.87 & 2.88 & 1.83 & -2.37 & 1.39 \\
22 & -0.07 & 0.01 & 0.00 & 0.00 & 0.00 & 0.04 & 0.01 & 0.00 & 0.00 & 0.00 \\
23 & -0.43 & 0.02 & 0.02 & 0.02 & -0.01 & 0.01 & 0.08 & 0.02 & 0.11 & 0.15 \\
24 & 1.32 & -0.13 & -0.13 & -0.18 & -0.15 & -0.33 & -0.13 & 0.05 & -0.18 & -0.16 \\
25 & -0.20 & -0.05 & 0.01 & -0.16 & 0.07 & -0.02 & 0.14 & 0.08 & 0.01 & 0.14 \\
27 & 0.23 & 0.09 & 0.01 & -0.05 & -0.12 & -0.11 & -0.10 & 0.08 & -0.04 & 0.00 \\
28 & 0.06 & 0.03 & 0.06 & 0.00 & -0.01 & -0.03 & -0.06 & -0.02 & 0.00 & 0.00 \\
29 & -0.11 & 0.01 & 0.01 & 0.01 & 0.01 & 0.02 & 0.01 & 0.01 & 0.01 & 0.05 \\
30 & 0.64 & -0.12 & -0.10 & -0.06 & -0.09 & -0.08 & -0.05 & -0.07 & -0.02 & -0.04 \\
31 & 0.00 & 0.00 & 0.00 & 0.00 & 0.00 & 0.00 & 0.00 & 0.00 & 0.00 & 0.00 \\
32 & 0.00 & 0.00 & 0.00 & 0.00 & 0.00 & 0.00 & 0.00 & 0.00 & 0.00 & 0.00 \\
33 & 0.00 & 0.00 & 0.00 & 0.00 & 0.00 & 0.00 & 0.00 & -0.01 & 0.00 & 0.00 \\
34 & -0.01 & -0.01 & -0.01 & 0.01 & 0.01 & 0.00 & -0.01 & -0.01 & 0.01 & 0.01 \\
35 & 0.00 & 0.00 & 0.00 & 0.00 & 0.00 & 0.00 & 0.00 & 0.00 & 0.00 & 0.00 \\
36 & 0.00 & 0.00 & 0.00 & -0.01 & -0.03 & 0.00 & 0.00 & 0.00 & 0.00 & 0.04 \\
\hline \hline
\end{tabular}

Channel systematic errors of $3 \mathrm{~K}$ can impose strong influence for this application. Band 28 is used to retrieve atmospheric profile variables, and a difference of $1.40 \mathrm{~K}$ in Channel 2 can cause severe uncertainty, which should be replaced or removed in data preprocessing. Band 29, combined with Bands 31 and 32, is usually used to retrieve surface temperature, but Channel 1 can introduce substantial error. Among the 16 MODIS TIR bands, only the channel errors in Bands 20, 22, 31, and 32 can be ignored.

\section{B. Channel Systematic Errors of Aqua MODIS Detectors}

The channel systematic errors analysis of Aqua MODIS employs data from June and December of 2003, which cover all of China and all are from the EOS data center. The results are shown in Table II. All bands except Band 21 are much more improved than Terra MODIS. Only Channel 1 of Band 24 has $1-\mathrm{K}$ systematic error. However, Band 21 has a large systematic error. Since it is mainly used in hot-spot detection, such large channel systematic errors can hardly be neglected, especially for small-scale hot spots. Consequently, it is necessary to combine with Band 22 when using Aqua MODIS data for fire detection. 

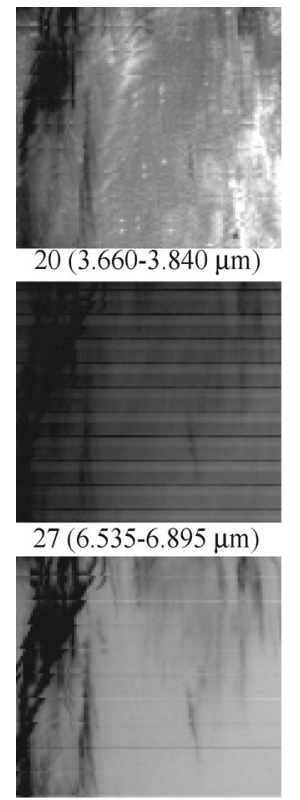

$33(13.185-13.485 \mu \mathrm{m})$
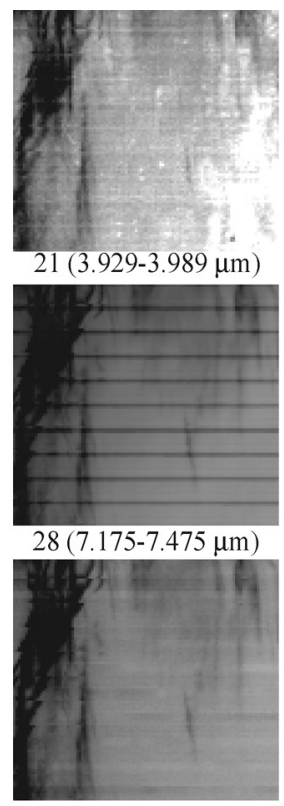

$34(13.485-13.785 \mu \mathrm{m})$
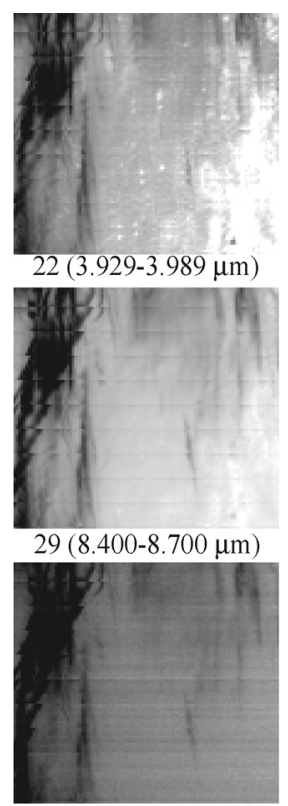

$35(13.785-14.085 \mu \mathrm{m})$
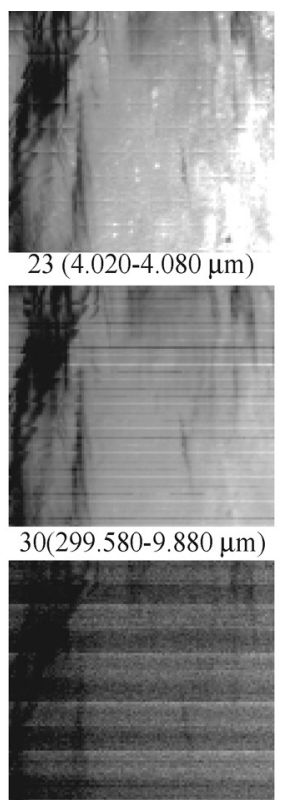

$36(14.085-14.385 \mu \mathrm{m})$
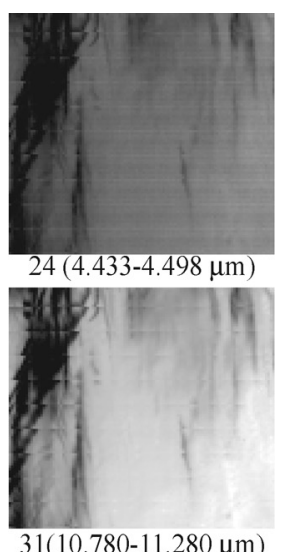

$31(10.780-11.280 \mu \mathrm{m})$

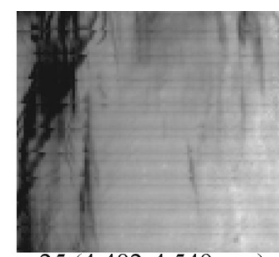

$25(4.482-4.549 \mu \mathrm{m})$

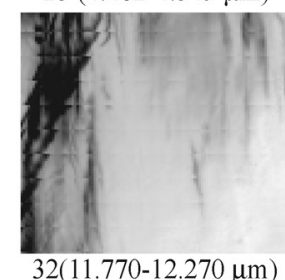

Fig. 1. Left-side images for Terra MODIS TIR band data. The images were received by the Beijing station on UTM 2:30 A.M. of August 15, 2002.

\section{Validation and Comparison}

Since it is difficult to directly validate the analysis results of channel errors, we use remote sensing data for the comparison of analysis results. Fig. 1 displays the part of MODIS TIR band images at Beijing time 10:30 A.M. on August 15, 2002. The images of Bands 20, 22, 31, and 32 are very smooth, which is consistent with the results in Table I. Band 21 contains abnormal strips, which demonstrates some evident errors in its channels and is consistent with the results in Table I. Bands 27,28 , and 36 have regular variation of strips, but the detector deviations are not very obvious because these strips are mainly from the mirror side effect [6]. The channels of Band 28 have an obvious line, which is caused by the strong deviation of Channel 2.

According to Wan [5], Channel 9 of Bands 21 and 22 and Channel 4 of Band 22 are too noisy to be used, while Channels $1-3$ of Bands $22-23,25$, and 27-30 contain strong systematic errors. However, our results are not all consistent with that. From Fig. 1, the image of Band 22 is as smooth as that of Band 31. It implies no interior error in that band, which coincides with our results. Because the L1B software for processing L1A to generate L1B data does not remove any error for a single detector [7], the data source should not be the reason for different results between these two methods. The possible causes of this inconsistency may be: 1) Wan's method does not remove the mirror side effect; 2) the land surface and/or atmosphere is not homogeneous enough to satisfy Wan's assumption; and 3) other noises may have a large effect on Wan's results because his samples are small.

\section{SUMMARY}

This letter analyzes huge amounts of MODIS data to determine the systematic error of TIR Bands 20-25 and 27-36. This method is based on the fact that some channels between two consecutive scans of MODIS data have overlapped pixels. It has different results from those of Wan [5]. For the analysis of Band 22, our results are more consistent with the visual effect of the images. This method is more robust and can cancel the mirror side effect compared with Wan's. Moreover, this method can be extended to process MODIS' other bands data that Wan's cannot do. The results show that channels of Bands 31 and 32 are very stable with systematic errors in all channels equal to $0.00 \mathrm{~K}$. Band 21, which is designed for the detection of hot spots, has strong systematic errors in both Terra and Aqua MODIS. Aqua MODIS data have better quality than that of Terra MODIS data except Band 21. Although these systematic errors would help to correct the detectors' systematic errors, they should not be used directly to do so. To achieve this goal, more details about the channel errors of detectors on observed radiance should be investigated.

Three major factors influence the results of our method, namely: 1) the assumption that the brightness temperature differences of the most overlapped pixels are randomly distributed, and these differences can be cancelled out for large data sample size; 2) surface elevation variation, which makes these most overlapped pixels deviate from the fixed locations so as not to meet the assumption in (1); and 3) high clouds make the most overlapped pixels deviate. Factor 2 is not obvious when elevation is low, and the influence from factor 3 can be excluded by cloud mask. Factor 1 is the basis of this method. The results from the analyses of four months of data in 2002, individually and as a whole, are quite similar, which renders the assumption acceptable when the data volume is large enough. Some other factors, such as hot spots and subpixel water, will result in a large deviation from our assumption. However, these occasional pixels can be negligible when large numbers of input samples are used. 


\section{ACKNOWLEDGMENT}

The authors would like to thank the anonymous reviewers for their insightful comments.

\section{REFERENCES}

[1] S. Liang, Quantitative Remote Sensing of Land Surfaces. New York: Wiley, 2004.

[2] I. J. Barton, "Satellite-derived sea surface temperatures: Current status," J. Geophys. Res.-Oceans, vol. 100, no. C5, pp. 8777-8790, 1995.

[3] Z. Wan, Y. Zhang, Q. Zhang, and Z. Li, "Validation of the landsurface temperature products retrieved from Terra Moderate Resolution Imaging Spectroradiometer data," Remote Sens. Environ., vol. 83, no. 1/2, pp. 163-180, Nov. 2002.
[4] W. L. Barnes, T. S. Pagano, and V. V. Salomonson, "Prelaunch characteristics of the Moderate Resolution Imaging Spectroradiometer (MODIS) on EOS-AM1," IEEE Trans. Geosci. Remote Sens., vol. 36, no. 4, pp. 1088-1100, Jul. 1998.

[5] Z. Wan, "Estimate of noise and systematic error in early thermal infrared data of the Moderate Resolution Imaging Spectroradiometer (MODIS)," Remote Sens. Environ., vol. 80, no. 1, pp. 47-54, Apr. 2002.

[6] B. W. Guenther, A. Wu, and X. Xiong, "Study of Terra MODIS reflective solar bands calibration stability and mirror side difference using simultaneous MODIS and MISR observations," Proc. SPIE, vol. 5652, no. 4, pp. 200-209, 2004.

[7] B. Guenther, X. Xiong, V. V. Salomonson, W. L. Barnes, and J. Young, "On-orbit performance of the Earth observing system moderate resolution imaging spectroradiometer: First year of data," Remote Sens. Environ., vol. 83, no. 1/2, pp. 16-30, Nov. 2002. 